\title{
V379 Cep: a System of Two Binaries
}

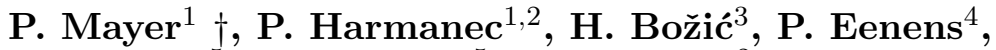 \\ E.F. Guinan ${ }^{5}$, G. McCook ${ }^{5}$, P. Koubský ${ }^{2}$, D. Ruždjak ${ }^{3}$, \\ D. Sudar ${ }^{3}$, M. Šlechta ${ }^{2}$, M. Wolf ${ }^{1}$, and S. Yang 6 \\ ${ }^{1}$ Astronomical Institute of the Charles University, Faculty of Mathematics and Physics, \\ V Holešovičkách 2, CZ-180 00 Praha 8, Czech Republic \\ ${ }^{2}$ Astronomical Institute of the Academy of Sciences, CZ-251 65 Ondřejov, Czech Republic \\ ${ }^{3}$ Hvar Observatory, Faculty of Geodesy, Zagreb University, 10000 Zagreb, Croatia \\ ${ }^{4}$ Departamento de Astronomia, Universidad de Guanajuato, 36000 Guanajuato, GTO, Mexico \\ ${ }^{5}$ Department of Astronomy and Astrophysics, Villanova University, Pennslyvania 19085, USA \\ ${ }^{6}$ Physics \& Astronomy Department, University of Victoria, PO Box 3055 STN CSC, Victoria, \\ BC, V8W 3P6, Canada
}

\begin{abstract}
We obtained new series of electronic spectra and $U B V$ photometry of V379 Cep and analyzed them in an effort to check whether the conclusion about its anomalous masses is not premature. We found that the second system of spectral lines seen in the spectra of V379 Cep does not belong to the secondary but to the primary of another binary $\mathrm{Ba}+\mathrm{Bb}$ (orbital period of $159^{\mathrm{d}} .0$ ) which constitutes a quadruple system with the narrow-lined $99^{\mathrm{d}} .76$ eclipsing binary $\mathrm{Aa}+\mathrm{Ab}$ (for which we report probable detection of the secondary and derive improved period and orbital elements). The mutual motion of the two binaries $\mathrm{A}+\mathrm{B}$ around the common centre of gravity is also observable and the probable orbital period is some 7878 days. Our result shows that the components $\mathrm{Aa}$ and $\mathrm{Ba}$ are somewhat evolved but removes the reported discrepancy of anomalously small masses. We find that V379 Cep is an astrophysically interesting quadruple system for which a future combination of interferometry, spectroscopy and photometry can provide individual masses of all four bodies.
\end{abstract}

Keywords. binaries: eclipsing, stars: early-type, stars: individual (V379 Cep)

\section{Introduction}

As found by Jerzykiewicz (1993) and confirmed by Clayton (1996), the variable star V379 Cep (also HD 197770, HR 7940) is an eclipsing binary; the spectral type is B2 IV, magnitude $V=6.3 \mathrm{mag}$, the period is 99.76 day. The minima are only about $0.05 \mathrm{mag}$ deep, the secondary minimum comes at phase 0.5 . Variability of radial velocity of this object has been known for a long time. Gordon et al. (1998) obtained modern spectra of this object and found two sets of lines: sharp lines and broad lines, both of identical spectral type. Gordon et al. interpreted them as lines of the primary and secondary components of the eclipsing binary; however, the resulting masses came out as rather strange, being only about a third of the values expected for the given spectral type.

\section{New spectroscopy and the second binary}

We obtained more spectra with the Ondřejov Observatory 2-m telescope (partly using HEROS, an echelle fiber spectrograph loaned by Landessternwarte Heidelberg) and the Dominion Observatory 1.2-m telescope. These data showed that the original interpretation was not true: the broad lines vary in RV but not with the $99^{\mathrm{d}} .76$ eclipsing-binary

$\dagger$ E-mail address: mayer@mbox.cesnet.cz 


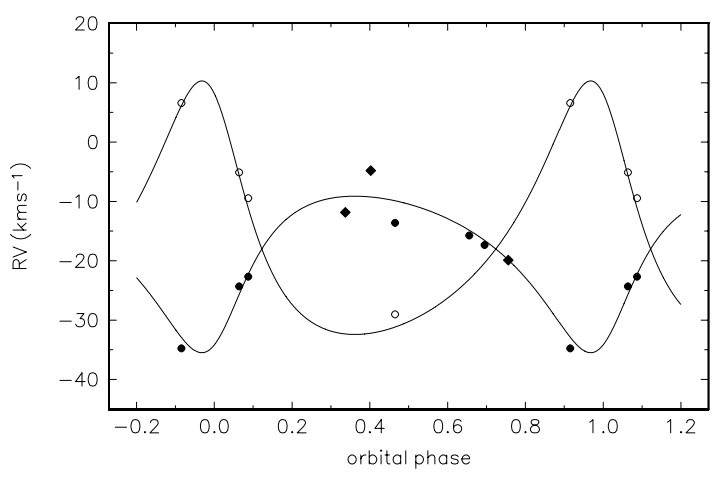

Figure 1. A phase diagram for the wide orbit with a period of 7878 days, as defined by the locally derived systemic velocities for the $99^{\mathrm{d}} .76$ and $159^{\mathrm{d}} .0$ binaries. The values from the old data are shown by diamonds, those for the new ones by circles (black ones for the $99^{\mathrm{d}} .76$ binary and empty ones for the $159^{\mathrm{d}}$ binary).

Table 1. Orbital parameters

\begin{tabular}{cccc}
\hline Element & Binary A & Binary B & Mutual orbit \\
\hline Peroid (days) & 99.7634 & 159.0 & $7878(?)$ \\
$T$ (periastron) & 2441905.52 & 2453004.5 & 2453090 \\
Eccentricity & 0.120 & 0.50 & 0.36 \\
$\omega\left(^{\circ}\right)$ & 268.1 & 58 & 206 \\
$K_{1}\left(\mathrm{~km} \mathrm{~s}^{-1}\right)$ & 43.2 & 18 & 12.8 \\
$K_{2}\left(\mathrm{~km} \mathrm{~s}^{-1}\right)$ & $75(?)$ & - & - \\
$A_{3} \sin i(\mathrm{AU})$ & 0.39 & 0.23 & 19 \\
\hline
\end{tabular}

period. Therefore, they belong to another binary. After collecting enough spectra, we were able to find a period for this binary of 159 days. It also became clear that the systemic velocities of both binaries vary in antiphase, i.e., both binaries revolve around the common mass centre. A possible period for this orbit is 7878 days, however this period and velocity amplitudes are not yet known with certainty, since the period depends upon velocities obtained early in the last century at Mount Wilson and DAO observatories (which we interpret as velocities of the sharp lines).

\section{Parameters of three orbits}

The orbital parameters of all three orbits are listed in Table 1, and the radial velocity curves are shown in Figures 1 and 2. Phases calculated relative to the periastron are used (also in Figure 3); note that then the phase of the primary minimum is close to 0.5 and of the secondary minimum to 0.0 .

\section{Light curve solution}

We obtained also additional photometry at the Hvar Observatory, San Pedro Martir Observatory and with APT Villanova. We found that the periastron longitude of the orbit of $\mathrm{A}$ is very close to $270^{\circ}$, which explains why the secondary minimum is so close to phase 0.5 (if the phase of the primary minimum is assumed zero). The photometry has not yet defined both minima with the accuracy needed for a reliable solution of the light 

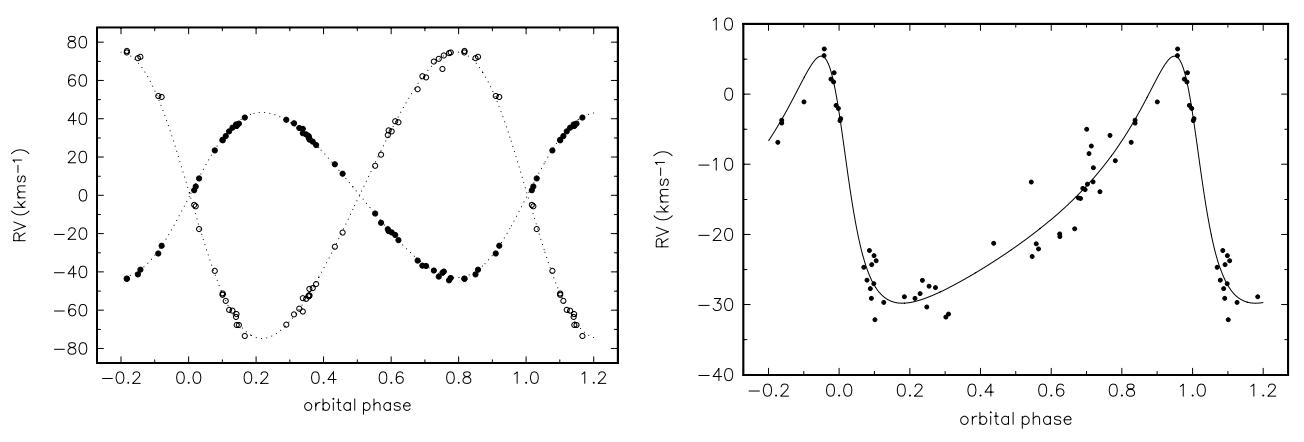

Figure 2. A phase diagram for the $99^{\mathrm{d}} .762$ (left) and $159^{\mathrm{d}}$ orbits (right) and all new RVs based on a FOTEL triple-star solution, i.e., prewhitened for the $7878^{\mathrm{d}}$ period.
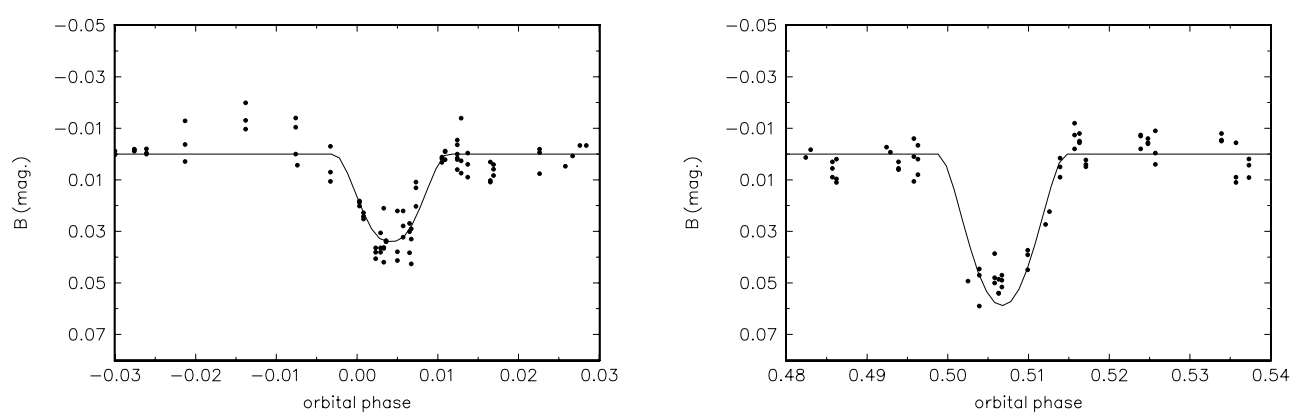

Figure 3. The $B$ light curves of the eclipsing system Aa+Ab with the solution for a $64 \%$ contribution of the light from B binary shown in the neighbourhood of both minima.

curve, but some preliminary results are possible. The best solution was obtained when the contribution of the binary B was assumed as 64\%; the observations are compared with theoretical light curve in vicinity of both minima in Figure 3. The secondary star in the eclipsing binary $(\mathrm{Ab})$ is of low luminosity (less than 1/10 of $L_{1}$ ), therefore its lines are invisible. Attempts to disentangle the spectrum around the line $\mathrm{H}_{\alpha}$ using KOREL (Hadrava 1995, 1997, 2004) gave some results, but — due to the low luminosity of Ab — only of low weight.

\section{Masses}

Very probably, all components of both binaries are normal stars, albeit the discussion of their masses is not yet quite unambiguous. Masses of the components of the eclipsing binary depend on the KOREL solution; with the present values, the mass of the primary component Aa well corresponds to its spectral type B2 IV. For the orbit of B, the mass function is $0.056 \mathrm{M}_{\odot}$, which means that the minimum mass of the secondary $(\mathrm{Bb})$ is less than $2 \mathrm{M}_{\odot}$. The total mass of the B binary follows from the mutual orbit; the mass of the primary $(\mathrm{Ba})$ is then about $9 \mathrm{M}_{\odot}$, again not too far from the value expected for the given spectral type (identical to Aa). 
Table 2. Binary A: FOTEL light curve solution and absolute parameters

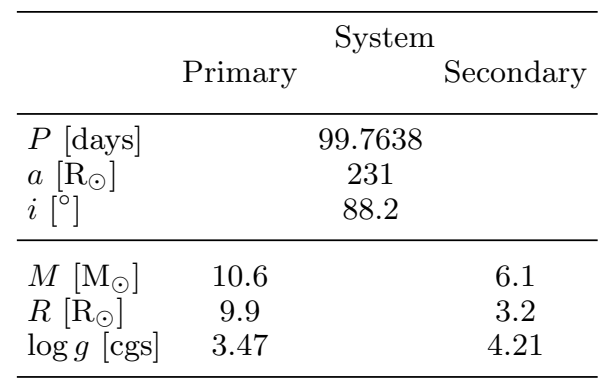

\section{Conclusion: interferometry needed}

The distance of the system was estimated as $440 \mathrm{pc}$ by Gordon et al. (1998). Due to the multiplicity, the distance is larger, and may be about $500 \mathrm{pc}$. The semimajor axis of the binary $\mathrm{A}$ is then 2.1 mas, of $\mathrm{B}$ is $2.5 / \sin i_{\mathrm{B}}$ mas. It will take several years until a better coverage of the mutual orbit is obtained. This orbit is so large that interferometry should separate both binaries - the advantage is that their magnitudes are comparable. The semimajor axis might be about 40 mas; at present however the binaries are close to conjunction, i.e., the separation depends mostly on the inclination of the orbit. Since integral masses of both binaries are known, this inclination can be calculated as $73^{\circ}$, and the separation should be under 20 mas for several next years. Both individual binaries might be separated by an advanced interferometer; the luminosity ratio is high in both cases, however.

\section{Acknowledgements}

We thank Drs. M. Jerzykiewicz and G.C. Clayton who provided us with their unpublished photometric observations. We acknowledge the use of the programs FOTEL, KOREL and PREKOR, made available by their author, Dr. Petr Hadrava. This research was supported from the grants GA ČR 205/03/0788 and GA ČR 205/06/0304 of the Czech Science Foundation and also from the research project AV0Z10030501 of the Academy of Sciences of the Czech Republic. We acknowledge the use of the electronic bibliography maintained by NASA/ADS system and by CDS in Strasbourg.

\section{References}

Clayton, G.C. 1996, PASP 108, 401

Gordon, J.A., Clayton, G.C., Smith, T.L. et al. 1998, AJ 115, 2561

Hadrava, P. 1995, A\&AS 114, 393

Hadrava, P. 1997, A\&SAS 122, 581

Hadrava, P. 2004, Publ. Astr. Inst. Acad. Sci. Czech Rep. 89, 1 \& 15

Jerzykiewicz, M. 1993, A\&AS 97, 421 